Thomas Diette, University of Lille, France

DOI:10.17951/Ismll.2020.44.4.79-90

\title{
Le réel à l'épreuve de la fiction dans Khalil de Yasmina Khadra
}

\author{
Reality vs. Fiction in Khalil by Yasmina Khadra
}

\begin{abstract}
RÉSUMÉ
Dans Khalil, Yasmina Khadra dresse le portrait réaliste d'un terroriste. Ce roman, écrit en 2018 et élaboré à partir d'un fait réel brouille les espaces du réel et de la fiction pour dévoiler les pensées apparemment inavouables d'un djihadiste. C'est cette stratégie du détour que cet article tâchera à mettre au clair. Il s'agira d'étudier comment Yasmina Khadra organise un entremêlement de ces deux mondes, l'espace fictionnel et l'espace réaliste, situés à la lisière l'un de l'autre, pour les faire interagir, se confronter mais aussi se questionner.

Mots-clés : Yasmina Khadra, Khalil, Djihadisme, pouvoir de la fiction, connaissance
\end{abstract}

\begin{abstract}
In Khalil, Yasmina Khadra paints a realistic portrait of a terrorist. This novel written in 2018 and developed from a real fact, blurs the spaces of reality and fiction to reveal the apparently unavowable thoughts of a jihadist. It is this detour strategy that this article will seek to clarify. It will be a question of studying how Yasmina Khadra organizes a scrambling of these two worlds, the fictional space and the realistic space, located at the edge of each other, to make them interact and confront each other but also to question.

Keywords: Yasmina Khadra, Khalil, Jihadism power of fiction, knowledge
\end{abstract}

\section{Introduction}

Nombreux sont les romanciers qui ont entrepris d'écrire sur le terrorisme des musulmans intégristes et Yasmina Khadra parmi les premiers. Dès la fin des années 1990, Les Agneaux du Seigneur (1998) puis À quoi rêvent les loups (1999) s'inscrivaient dans cette thématique que L'Attentat, en 2005, prolongera avec succès. Toutefois, le problème soulevé par Khalil, sorti en 2018 est d'une nature différente. Dans cette fiction, nourrie d'un épisode réel, les attentats au Stade de France le 13 novembre 2015, on découvre l'islamisme religieux à l'intérieur d'un décor européen. Le romancier scrute l'actualité pour en donner une vision nouvelle permettant ainsi à la composante fictionnelle une vue au-delà de l'anecdote.

Khadra réagit à l'instantanéité de l'Histoire en s'emparant d'un sujet brûlant mais s'il puise dans le vivier des épisodes sanglants du djihadisme en Europe,

Thomas Diette, Université de Lille, Domaine Universitaire du Pont de Bois, 3 Rue du Barreau 59650 Villeneuve-d'Ascq, thomas.diette@gmail.com, https://orcid.org/0000-0002-7090-2179 
il ouvre le champ des possibles en se demandant comment les kamikazes en sont arrivés à commettre leurs exactions. Le lecteur est placé en première ligne de ce qui pourrait représenter l'horreur en son acmé et raviver chez lui des souvenirs tragiques. Toutefois, le roman ne se limite pas à un simple rappel de ces événements. La fiction s'inspire de ces faits réels pour s'en détacher. Cette distanciation est le propre de l'écrivain, comme le souligne Garand,

Proposer une littérature de qualité, susciter la réflexion, déjouer les préjugés en jetant un regard nuancé sur les phénomènes de manière à faire saisir toute leur complexité, accomplir tout cela de surcroît au moyen de romans accessibles et palpitants, tel semble être le défi que s'est lancé Khadra (Garand, 2008, p. 41).

Contrairement à L'Olympe des infortunes, fable humaniste publiée en 2010 qui se déroulait dans un non-lieu et dans un temps non identifiable, le roman est ici en prise directe avec le lecteur. L'action commence in medias res par l'arrivée de Khalil et des autres soldats de Dieu pour semer le chaos dans la capitale. Sans apporter de réponse formelle, la fiction retisse le parcours du jeune homme et lui propose un nouveau destin. De fait, il est engagé dans un nouveau questionnement : et si sa nouvelle famille, la minorité islamiste qu'il a rejoint, lui avait menti sur son rôle dans ces attentats ? Et s'il s'était fourvoyé depuis le début de son embrigadement ? Et si la voie de la reconnaissance passait par d'autres chemins?

\section{L'anecdote à la lumière de la fiction}

Si Khadra choisit ce sujet sulfureux, c'est autant dans une visée militante, en adoptant la posture de l'écrivain armé de sa plume qui lutte contre le fanatisme, que dans une visée ontologique pour mettre à nu des pensées apparemment inavouables en public. Le recours à l'écriture des maux est un moyen pour Khadra de dire ce qui ne pouvait être dit et confère une portée plus grande aux événements relatés. L'accès à une conscience paranoïaque est un procédé déjà utilisé dans La Dernière Nuit du Raïs, en 2015, dans lequel Khadra avait infiltré la tête de Kadhafi. Ici, la fiction prend encore plus d'ampleur en dévoilant les rouages de la pensée torturée d'un kamikaze imaginaire hanté par son échec. Le romancier réinvestit la figure du Mal pour fouiller la noirceur des Hommes tout en racontant des personnages hautement romanesques.

Pour Lasnari ${ }^{1}$, dans un ouvrage consacré à la «trilogie du malentendu » de Khadra, l'événement est un pré-texte à la fiction. C'est le point de départ duquel le récit peut naître, non sans le transformer. Tel un reportage en immersion,

1 C'est Khadra qui a nommé lui-même l'ensemble constitué de L'Attentat, Les Hirondelles de Kaboul et Les Sirènes de Bagdad sous le titre de trilogie du malentendu. L'auteur se réfère à des faits historiques contemporains pour montrer la fracture entre l'Orient et l'Occident. 
le lecteur suit de l'intérieur l'action du premier commando entré en opération près du Stade de France, à l'occasion d'un match amical de football opposant la France à l'Allemagne. Dans les premiers chapitres de Khalil, la frontière entre l'imaginaire et le réel est floue. En effet, Khadra se sert du canevas de l'anecdote réelle pour lancer sa fiction. Mais inversement, la fiction nourrit aussi l'anecdote car elle lui donne une vue d'ensemble. Elle déchiffre, lui donne une origine et une potentialité, certes romanesque, mais qui tient du vraisemblable. Cette puissance heuristique du roman ouvre la voie à un champ de référence de second degré, un champ des possibles, comme l'a défini Paul Ricoeur dans Temps et Récit mais aussi dans la préface de La Métaphore vive ${ }^{2}$. Pour le dire autrement, la fiction est une réinterprétation du réel. Et si la scène s'était produite de cette façon...

L'incipit met en scène cinq hommes, parmi lesquels Ali, le conducteur et quatre kamikazes dont trois mourront, excepté le narrateur. Pourtant, même si leur mission doit coordonner des attentats-suicides successifs dans le stade et à sa périphérie, l'écrivain reprend à son compte les plans réels des terroristes. Deux d'entre eux (inconnus de Khalil dans la fiction) s'apparentent aux deux Irakiens qui ont été les premiers à déclencher les hostilités aux abords du stade : «Il y avait deux frères que je ne connaissais pas, un devant avec Ali le chauffeur, l'autre sur la banquette arrière à côté de Driss, et moi » (Khadra, 2018, p. 11). Le troisième est Driss, l'ami d'enfance et le compagnon de route de Khalil dont la mort sera relatée plus loin dans le récit : «Ton pote, il a été identifié. Il fait la une des JT, ce matin. C'est un des kamikazes du Stade de France » (p. 11). Khadra reprend ainsi la trame de l'épisode réel, avec les trois explosions successives et les trois djihadistes morts. Le narrateur doit interpréter les événements à l'aune de sa connaissance du plan global : « les 'explosions' [....] programmées à l'intérieur du Stade de France seraient donc nos deux inconnus » (Khadra, 2018, p. 30, les guillemets sont dans la version originale). L'éloignement spatial du narrateur, relégué dans la station de RER pour commettre son action suicide rend sa perception très incertaine.

Le roman n'est pas la simple reproduction du réel mais bel et bien une «modélisation du l'univers réel » pour reprendre l'expression de Genette ${ }^{3}$. L'œuvre littéraire reprend à son compte les éléments référentiels pour en augmenter la puissance signifiante. La fiction est bien une création, mais c'est une invention régie par une logique différente que celle du monde référentiel, d'après John Searle dans son ouvrage Les Actes du langage:

\footnotetext{
2 Ricoeur y parle d'une «stratégie du discours qui, en préservant et développant la puissance créatrice du langage, préserve et développe le pouvoir heuristique déployé par la fiction » [italiques de l'auteur cité] (Ricoeur, 1975, p. 10).

3 Genette (1983) n'en conclut pas pour autant que la fiction vise à tromper le lecteur. L'œuvre littéraire est le produit de la toute-puissance créatrice de l'auteur qui vise in fine à transmettre des messages marquants. Cette approche permet une prise de distance de la stricte réalité.
} 
La fiction est un acte de langage transgressif qui ne se développe pas selon la logique verticale par laquelle on dit le monde, celle du monde référentiel, mais selon une logique horizontale qui serait coupé de la dynamique référentielle (Searle, 2009, p. 165).

Entre le littéraire et le non-littéraire, c'est un jeu d'échos et de résonnances qui se répète. C'est pour cette raison que l'écrivain situe le cadre de l'enfance de Khalil dans le quartier bruxellois de Molenbeek. L'allusion à ce foyer djihadiste donne du crédit à son récit en référant à ce lieu explicitement lié par les médias au communautarisme et à l'islamisme radical. De manière analogue, la ceinture d'explosifs défectueuse pourrait faire référence à celle de Salah Abdeslam, connu pour son implication dans les attentats terroristes du 13 novembre 2015 et pour sa cavale de 125 jours. L'écrivain fusionne en quelque sorte plusieurs épisodes en rapport avec des kamikazes pour donner plus de force à son récit. Il appelle le lecteur à tisser des liens avec des faits médiatiques pour assurer une continuité entre sa fiction et des événements connus. Le roman repose alors bien sur des bases vraies ${ }^{4}$.

Pourtant, des discordances sont perceptibles. Le roman suit bien la trame initiale mais s'en éloigne rapidement. Il n'a jamais été question d'un autre kamikaze composant ce premier commando. Khalil est ainsi un pur personnage de fiction. Il doit découvrir pourquoi sa ceinture a failli et comment mener sa mission à bien dans ces conditions. Le lecteur entre en contact immédiat avec ce personnage qui fait son autopsie de kamikaze. Le roman apparait donc comme une suite fictive d'un événement vrai. Dans un article de la Nouvelle Revue Française intitulé « Le roman, un mal nécessaire ? », Roy (1968) développe l'idée que ce que les histoires imaginaires nous donnent, c'est peut-être la véritable histoire de la vie réelle.

\section{Une représentation des chemins brisés de l'enfance}

La matière romanesque peut se nourrir des apories du réel et inventer un passé et un avenir à ses protagonistes. Elle part d'une tragédie collective pour arriver à la représentation d'un drame individuel face aux circonstances de l'Histoire. C'est par un retour rétrospectif sur sa vie que peut se comprendre le tournant religieux du narrateur. Le récit de l'enfance de Khalil commence une fois la mort repoussée à plus tard. En effet, le jeune homme a failli dans sa mission. Il n'aura pas réussi à tuer un maximum de personnes puisque sa ceinture d'explosifs ne s'est pas déclenchée. Il est condamné à faire toujours partie du monde des vivants, contrairement à son ami d'enfance Driss qui, lui, a mérité son statut de martyr. Khalil, qui peut être traduit de l'arabe par « le bien-aimé » est-il responsable de son malheur?

4 Barthes (1968) rappelle dans son essai «L’Effet de réel » que le réel est la référence essentielle dans le récit historique. L'histoire, aussi récente soit-elle, s'appuie non pas sur le vraisemblable mais sur ce qui est vraiment arrivé. Toutefois, Khalil n'est pas à proprement parler un « récit historique » mais un roman qui s'inspire d'un fait vrai. 
En imaginant un quatrième terroriste au Stade de France, Khadra donne voix au kamikaze qui relate à la première personne sa conception du monde. Pivot et filtre subjectif de la narration, le roman lui construit un passé marqué par l'absence de cadre autoritaire dans la maison familiale. A l'inverse de son ami Rayan, qui a pu compter sur une mère aimante et une éducation héritée des traditions berbères, Khalil et son frère d'adoption Driss sont des êtres privés de repères. Le récit montre que la fracture du kamikaze est lointaine, aussi ancienne que son enfance dénuée de modèle :

Mon père n'avait jamais jeté un œil sur mes bulletins, ornés pourtant de notes catastrophiques. Il préférait picoler et se ruiner au tiercé. Quant à ma mère, analphabète, elle était incapable de distinguer une facture d'une convocation. En réalité, à la maison, tout le monde s'en foutait (Khadra, 2018, p. 58).

Le terroriste est donc un individu qui a déjà un problème sous le toit parental. Il ne trouve pas les fondations qui pourront soutenir l'adulte en devenir. C'est surtout sur le personnage du père que vont se cristalliser les reproches du fils :

La morale n'était pas le rayon de mon père. En apprenant que j'avais redoublé la sixième, il avait fait claquer la langue contre son palais et dit sur un ton qui résonnerait longtemps en moi : " Même avec une selle brodée sur le dos, un âne restera un âne ». Je m'attendais à un sermon dans les règles ou bien à une leçon de vie légendée d'exemples frappants et de noms de personnes parties de rien devenues célèbres et riches grâce à leur dévouement à l'école, enfin à des paroles censées m'éveiller à mes responsabilités ; je n'eus droit qu'à un mépris cinglant (Khadra, 2018, p. 75).

La métaphore religieuse du sermon prend ici tout son sens quand il s'agit de trouver une famille de substitution composée de musulmans intégristes. L'idéologie de l'islamisme attire les gens vulnérables. Le roman s'ingénie à reconstituer le fil de la conversion à cette idéologie mortifère. La voix de l'intérieur donne une portée insoupçonnée à cette adhésion perçue comme une planche de salut pour le jeune homme.

Prenant pour modèle Lyès, son chef de guerre, le narrateur raconte que l'adhésion au fanatisme s'explique avant tout par cette construction identitaire minée dès le plus jeune âge :

À l'époque, l'adolescent Lyès n'avait ni dieu ni prophète. La religion lui était aussi étrangère que ces formules mathématiques qui vous court-circuitent les neurones avant que vous ayez fini de les recopier sur le cahier. Il n'était qu'un mal luné de dix-sept ans qui ne savait rien faire de ses dix doigts, à part mettre son poing dans la figure d'un gars de la cité d'en face ou bien montrer son majeur à un vigile trop curieux. [...] Eh bien, tout ça était fini. Kamis et barbe rougie au henné, Lyès avait trouvé sa voie et occupait le rang d'émir (Khadra, 2018, pp. 12-13).

Dès lors, Khalil peut constater qu'il est devenu un étranger au sein de sa propre famille. Mis à l'écart à son retour de France, par sa sœur aînée, Yezza, c'est 
auprès de sa sœur jumelle Zahra que le narrateur parvient à garder l'unique lien affectif. Celle-ci est une figure de la réconciliation. Elle l'encourage notamment à reconsidérer les valeurs de l'islam. Selon elle, Dieu incite à renouer les liens avec son géniteur : "L'islam prône le pardon. La piété filiale est aussi sacrée que la piété elle-même » (Khadra, 2018, p. 159). Mais face à un nouveau refus du jeune homme, elle disparaît à son tour, et mourra peu de jours après dans un attentat terroriste dans le métro bruxellois. Par cet effet romanesque, elle devient en quelque sorte une victime collatérale d'une action suicide qu'aurait pu perpétrer Khalil. Khadra souligne par ce jeu de coïncidences que le djihadisme est l'affaire de tous et que personne ne peut se croire en sécurité face au danger qu'il représente.

\section{Le djihadisme de l'intérieur par l'œil du romancier}

Le réel ne pouvant être retranscrit fidèlement, il n'apparaît dans la fiction que sous la forme d'un analogon et y est donc hors d'atteinte. C'est donc dans l'œuvre et dans elle seule que les « réseaux signifiants $»^{5}$ agissent. Khalil propose au lecteur l'exemple d'un terroriste plutôt surprenant. Alors même que l'on pourrait s'attendre à un kamikaze muré dans ses certitudes, le romancier nous donne paradoxalement un aperçu d'une psyché contrariée. En effet, le roman suit à la première personne du singulier le parcours du héros éponyme confronté à une remise en question de toutes ses convictions. Le choix de la narration homodiégétique permet au récit de mettre à nu la fragilité du jeune homme.

Le récit reconstitue le drame intérieur de cet être de papier. Il met surtout en relief l'angoisse du personnage, plongé dans une interrogation destructrice. Khadra exploite la focalisation interne pour décrire la lente mais néanmoins brutale descente de Khalil dans les profondeurs de l'incertitude. Ce dernier n'a de cesse de constater qu'il n'est qu'une pièce dans la grande machinerie de l'idéologie islamiste. Il représente la recrue idéale pour la cause djihadiste : pour lutter contre son état d'insatisfaction permanente, il a été accueilli dans les bras bienveillants de ses frères qui lui ont donné une seconde chance. Il pense pouvoir enfin devenir quelqu'un d'important mais pour y parvenir complètement, il doit devenir un martyr. Il défend alors tous ces coreligionnaires morts pour leur cause, refusant qu'on les considère comme des détraqués. De façon conventionnelle, le roman exploite ainsi les stéréotypes en matière d'embrigadement ${ }^{6}$.

5 La sociocritique lancée par Duchet au début des années 1970 évoque l'autosuffisance du microcosme romanesque. La fiction dépasse son statut de simple reflet, elle devient un monde autonome. Dans cette optique, Khalil réfère bien à des réalités sociales mais pour décoder les discours sur celles-ci et ainsi interroger un univers de signes qui lui est propre (Duchet, 1974).

6 Le roman met en évidence l'appartenance à la religion musulmane du narrateur qui sépare « nous » d'un côté et « les autres » d'autre part. Boraso (2019) explique que cette scission engendre chez le lecteur occidental un sentiment d'hostilité qui perdure jusqu'à la fin du récit. 
Mais l'originalité du roman tient au fait que le romancier décrit d'abord et avant tout un garçon à qui la société occidentale n'a pas permis de nourrir ses espoirs. Il est alors lui-même la victime d'un système qui le dépasse. Le processus de radicalisation est décrit comme un chemin de traverse emprunté sans y prêter attention. L'enchaînement des choix qui mènent à l'intégrisme est résumé par Khalil lors d'un dialogue avec Moka, vieux bougre qui erre dans leur quartier d'enfance : «Il suffit de bien peu de choses pour que l'on dégringole dans l'estime de soi. Et alors, bonjour les dégâts. Tout part en vrille » (Khadra, 2018, 124). C'est dans les dernières pages du roman que le jeune homme raconte comment il a changé pour être embrigadé. C'est un enrôlement qui se produit de façon subreptice, involontaire et implacable. Au commencement, le sujet se décrit comme un être insatisfait mais sans rancune contre le mauvais sort :

Je n'en voulais à personne. L'existence est ainsi faite ; il y a des gens aisés et des gens lésés, des gens à qui tout réussit et des canards boiteux. Bien sûr, au début, je cherchais à comprendre pourquoi la chance ne me souriait pas. Je me posais un tas d'autres questions, sauf que les réponses bottaient en touche. À la longue, je ne me prenais plus la tête (Khadra, 2018, p. 199).

Mais sans le désirer, il va être happé par le pouvoir hypnotisant de la rhétorique des imams :

Jusqu'au jour où, en suivant un reportage sur le djihad, tu entends : «Les mercenaires meurent pour leurs commanditaires. Les soldats pour des intérêts qui ne leur apportent rien. Les gangsters pour des prunes... Mais le chahid $^{7}$, lui, il ne meurt jamais ; il se prélasse dans les jardins du Seigneur, entouré de houris et d'arcs-en-ciel éblouissants [italiques de l'auteur cité] (Khadra, 2018, p. 200).

Finalement, tel un corps qui lutte contre une maladie et qui abandonne, la vampirisation se termine par le verbe enchanteur de l'imam :

Il a la réponse à toutes les questions qui te taraudaient autrefois sans te livrer un indice susceptible de t'éclairer; il te renvoie à tes déconvenues, aux vexations que tu croyais avoir surmontées, à tes blessures jamais cicatrisées - le paumé devient ton sosie, le révolté ton frère siamois, les prêches ton exutoire, la violence ta légitimité (Khadra, 2018, p. 201).

En montrant les étapes de la radicalisation de Khalil, Khadra nous fait découvrir les rouages de l'organisation par un de ses adhérents. La promesse d'être élevé et protégé dans cette nouvelle famille se heurte toutefois à un certain nombre de contradictions. Comme dans un foyer, une hiérarchie est instaurée dans le mouvement islamiste. À sa tête trône l'imam Sadek, l'autorité spirituelle. C'est un personnage lointain et proche à la fois, en ce sens qu'il donne des directives

$7 \quad$ [le martyr] 
par des intermédiaires. Par conséquent, Khalil lui obéit sans avoir affaire à lui directement. Il est secondé par Lyès, le cheikh, qui ordonne et guide les jeunes adeptes. Un djihadiste n'est donc pas un être libre. Il se doit d'être servile et de respecter ceux qui le commandent. Il n'a pas peur de mourir, ne craint pas de rompre ses liens avec sa famille de sang, mais il vit dans la peur constante de son émir. Khalil sait sa vie en danger s'il ne respecte pas un certain nombre de règles impérieuses. Lyès est une menace constante pour le jeune homme, notamment durant les interrogatoires qu'il mène pour savoir si ses "soldats » sont prêts à tout pour aller au bout de leur mission. Les entretiens entre les deux intégristes musulmans montrent l'ascendant du chef et la crainte qu'il inspire :

Le repli de Lyès n'était qu'un traquenard savamment camouflé. L'émir m'accordait le bénéfice du doute sans pour autant surseoir les soupçons. Je le connaissais trop bien pour prendre ses excuses pour argent comptant. Lyès n'était pas du genre à passer l'éponge ou à laisser quelque chose au hasard, encore moins à blanchir un repenti ou un accusé à tort. Lorsqu'il feignait de tourner la page, il en ouvrait aussitôt une autre en y reprenant les mêmes parenthèses que celles dûment consignées sur la précédente. Et quand il avait quelqu'un dans le collimateur, il enlevait le cran de sûreté et repliait son doigt sur la détente, certain de finir par tirer (Khadra, 2018, p. 218).

La méfiance du cheik à l'encontre de Khalil souligne bien à quel point le mensonge et la dissimulation sont à l'œuvre au sein de leur mouvement. Le kamikaze n'est qu'un simple exécutant, dépassé par des forces qui lui sont supérieures.

\section{Temps et espaces de la fiction}

Deux temporalités oscillent tout au long du récit. D'une part, le temps de l'urgence. Khadra se réapproprie les codes du roman policier ${ }^{8}$ grâce au rythme haletant d'une fiction qui place un personnage en cavale qui doit se cacher et dans le même temps chercher à comprendre ce qui s'est produit avec sa ceinture d'explosifs. Le héros doit trouver une échappatoire tout en enquêtant sur les raisons de l'échec de sa mission. L'ancrage historique donne son armature à la première partie du récit. Ce qui fait le centre de la narration, ce ne sont pas les attentats, mais tout ce qui arrive après. Le roman peut se lire comme une tentative sans cesse renouvelée de le faire changer d'opinion : c'est le rôle dévolu aux autres personnages. Mais ni le mépris de Rayan, qui clôt de façon dramatique la première partie du roman ni la mort inattendue de sa sœur jumelle ne parviendront à le faire vaciller a priori. Dans cette course contre la montre, jamais il n'est question d'un lendemain plus radieux.

D'autre part, le roman retrace le passé du personnage avec la reconstitution de sa (dé)construction identitaire, des étapes qui ont jalonné sa vie ab ovo. Le

8 Khadra connaît bien ce genre puisqu'il avait connu le succès dans les années 1990 grâce à la série des « Commissaire Llob». 
narrateur semble prisonnier d'une histoire personnelle douloureuse. Les souvenirs sont autant d'analepses ${ }^{9}$ sur les épisodes décisifs de l'enfance marqués par des privations et des échecs. Par un effet de cascade, la mise à l'écart de la cellule familiale a conduit à la barbarie. Le roman décrit cette chute en avant, que rien ne peut repousser. Le temps est ainsi un compte à rebours avant la mise à mort d'un nombre important d'inconnus. Ce temps, qu'il soit révolu, présent ou futur est ennemi ; la vie du personnage ne peut échapper au chaos. Pris au piège, chaque jour apporte son lot de haines et de combats, comme il en était déjà question dans les pages sanglantes de L'Attentat qui nous plongeait au cœur du conflit israélopalestinien. L'histoire de Khalil est le recommencement infernal des mêmes dynamiques viciées qui alimentent sa vie : il devra mener à bien une seconde mission pour devenir un martyr.

Alors que faire face à ce temps qui englue le jeune homme dans un réel voué à l'échec ? Peut-être reconsidérer la valeur de l'espace. Le retour aux sources est un élément majeur dans la structure de l'œuvre. Dans celle-ci, il y est toujours question d'appartenance. Un islamiste est souvent un apatride ou plutôt un étranger. Un dialogue avec un groupe de jeunes Maghrébins permet d'illustrer l'implacable logique de Khalil :

Élevez le niveau, cousins, dit-il doctement. Ce qui se passe est l'aboutissement logique d'un processus aussi vieux que l'instinct grégaire : l'exclusion exacerbe les susceptibilités, les susceptibilités provoquent la frustration, la frustration engendre la haine et la haine conduit à la violence. C'est mathématique (Khadra, 2018, p. 81).

C'est par le retour aux sources, la vision d'un lieu chéri, que se renversera le cours des choses. En effet, le narrateur revient au pays de ses ancêtres dans l'explicit du roman. C'est le cadre de sa nouvelle mission suicide. On lui ordonne de commettre un attentat suicide à Marrakech. C'est le pays des souvenirs heureux, celui des odeurs d'enfance, des visions à perte de vue sur les vergers et surtout des moments de complicité avec Zahra. Au Stade de France, Khalil se sentait investi d'une mission sur un territoire inconnu et étranger comme dans les croisades. Ici, son émir exige de lui qu'il sème le chaos dans un lieu qu'il aime. La dernière page du roman est assez énigmatique. L'on comprend que l'attentat a échoué quelques heures avant le passage à l'acte. Une carte postale écrite par Khalil et adressée à Rayan laisse entendre que l'échec de l'opération pourrait être dû au revirement du narrateur : « Moka n'avait pas tort. Le vrai devoir est de laisser vivre » (Khadra, 2018, p. 228). Bouveresse souligne que l'expérience de la limite est souvent un échec :

9 Selon Tazartez (2013), l'analepse dans les fictions du terrorisme constitue une tentative de structuration de ce qui a explosé. 
Si l'homme s'approche suffisamment de la limite, ce qu'il sera amené à faire presque fatalement à un moment ou à un autre, il aura la possibilité de se rendre compte que son espace n'est pas l'espace et que sa lumière n'est pas la lumière (Bouveresse, 2011, p. 129).

De façon surprenante, Khadra (2018) renonce donc à faire mourir le jeune homme en martyr. C'est sans doute que le charme des émirs n'agit plus lorsqu'on est revenu aux lieux du bonheur et lorsqu'on regarde la réalité en face. Manipulé à des fins idéologiques, le jeune homme avait perdu la liberté de conscience. Désormais, seule la fatalité compte selon lui et elle seule pourra expliquer les événements, aussi tragiques soient-ils. Cette vue de l'esprit le conduit à repenser le rôle des hommes sur terre. Ne subsistent que deux espaces : le Ciel, promesse faite aux croyants qui manifeste sa foi, et le monde sensible qu'il faut quitter le plus rapidement possible pour atteindre le paradis. Attardons-nous sur cette dialectique mise en valeur tout au long du récit : la confrontation entre l'aspiration à rejoindre promptement le paradis et notre responsabilité à vivre pleinement notre condition de mortel.

Khadra (2018) expose un combat intérieur qui tient en haleine le lecteur tout au long du roman. Khalil doit choisir : suivre les principes en vigueur dans le djihadisme et tourner le dos à tout ce qui touche à la vie terrestre ou désavouer ses idéaux et revenir dans le monde qui l'a happé depuis son enfance. Ce monde terrestre est perçu par le jeune homme comme vide et passager :

On aurait eu envie de tendre la main pour retenir ce qui s'enfuyait, mais je ne tendrais pas la main car rien ne m'importait plus que cette implacable vérité : tout, ici-bas, est éphémère, chimérique et vain... Ne restera, par-dessus les absences et les finitudes, que le visage du Seigneur [italiques de l'auteur cité] (p. 176).

Le futur martyr se voit alors comme un vengeur face aux individus impurs : « On ne peut pas redresser le monde sans le débarrasser de ceux qui courbent l'échine » (p. 83). Le jeune homme demeure insensible aux douleurs de ceux qui l'entourent. Il affiche une adhésion à toute épreuve :

On ne change pas d'un iota les convictions du vrai croyant. [... ] J'étais déjà ailleurs, inexpugnable dans ma tour flottante ; j'étais là où pas une illusion d'optique ne pouvait flouter mes repères de musulman. J'avais développé un rapport strictement cosmique aux êtres et aux choses [italiques de l'auteur cité] (p.175).

Contre les ombres et les souffrances du monde terrestre, le paradis et ses lumières sont offerts aux martyrs. Le héros est conduit vers ce choix par défaut semble-t-il car rien ne peut le retenir. Tout le pousse à espérer mieux car il est dans l'impossibilité de sortir des mailles inextricables du terrorisme. Le revirement final du narrateur apparaît en définitive comme une sortie de l'aveuglement forcé. Khadra illustre le refus de la fatalité et réaffirme sa foi inébranlable en le libre arbitre. 


\section{Conclusion : la fiction pour dépasser les stéréotypes}

Ce roman, fiction spéculative sur les maux de notre temps, interroge en profondeur le lecteur au sujet de sa perception du djihadisme ${ }^{10}$. Le récit transforme le fait réel, l'amplifie, le détourne et en fin de compte l'interroge au-delà des clichés. Khadra invite les lecteurs à se méfier des idéologies présentées comme des vérités absolues. Il n'épargne aucun acteur impliqué dans cet engrenage meurtrier, ni les parents qui se déresponsabilisent de l'éducation de leurs enfants, ni leurs progénitures qui n'ont plus la volonté de s'en sortir, ni les gouvernements qui ont laissé se creuser les disparités sociales, ni la société qui catégorise, divise et rejette, ni surtout les terroristes avides de pouvoir en profitant de la vulnérabilité d'une jeunesse désabusée et fragile.

On pourrait pousser l'idée plus loin en affirmant que le djihadisme est second dans le roman. La fiction tend à effacer le fanatique en montrant plutôt l'homme qui se cache derrière. C'est en définitive la question de la quête de vérité(s) qui prédomine. Cette dynamique traverse toute l'œuvre de Khadra (Abouali, 2013) ${ }^{11}$ Comment admettre une autre vérité quand l'unique voie possible était celle de Dieu?

Khalil est bel et bien une fresque des ruptures sociales dans les communautés occidentales mais c'est aussi un roman de la subjectivité qui peint le drame intérieur d'un personnage. Khadra, au fil d'un récit suffoquant, nous conduit à nous interroger sur l'accomplissement de nos destinées. Dans la nuit de Khalil, une lueur d'espoir surgit : il est en mesure de décider d'être l'artisan de son bonheur ou le responsable de son malheur. La lecture du roman invite à l'optimisme car ce choix paraît possible : l'écrivain montre que nous sommes tous maîtres de notre destin et pouvons quand nous le désirons modifier la donne de départ.

\section{References}

Abouali, Y. (2013). Yasmina Khadra ou la recherche de la vérité. Paris: L'Harmattan.

Barthes, R. (1968). L'effet de réel. Communications, 11, 84-89.

Boraso, S. (2019). Yasmina Khadra. Khalil. Il Tolomeo, 21, 317-321.

Bouveresse, J. (2011). Que peut-on faire de la religion? Marseille: Agone.

Duchet, C. (1974). Le Réel et le texte. Paris: Armand Colin.

Garand, D. (2008). Que peut la fiction ? Yasmina Khadra, le terrorisme et le conflit israélopalestinien. Études françaises, 44(1), 37-56.

Genette, G. (1983). Nouveau Discours du récit. Paris: Seuil.

Kadari, L. (2007). De l'utopie totalitaire aux ouvres de Yasmina Khadra, approche des violences intégristes. Paris: L'Harmattan.

Khadra, Y. (2018). Khalil. Paris: Pocket.

10 Schaeffer (1999) dans son essai Pourquoi la fiction? affirme que la fiction a une valeur cognitive. Il s'agit d'une modalité de la connaissance par « modélisation cognitive du réel ».

11 Cette thématique a été mise en valeur dans la « trilogie du malentendu » de Khadra par Abouali (2013). 
Lasnari, A. (2016). La trilogie du malentendu de Yasmina Khadra. Paris: Publisud.

Ricoeur, P. (1975). La Métaphore vive. Paris: Seuil.

Roy, C. (1968). Défense de la littérature. Paris: Nouvelle Revue Française.

Schaeffer, J.-M. (1999). Pourquoi la fiction? Paris: Seuil.

Searle, J. (2009/1972). Les Actes du langage, Paris: Herman.

Tazartez, C. (2013). Fictionnalisation de l'attentat-suicide : surexposition d'un triple dispositif chez De Lillo, Binebine et Khadra. TRANS- (online), 15. Retrieved November 25, 2020, from http:// journals.openedition.org/trans/781. 